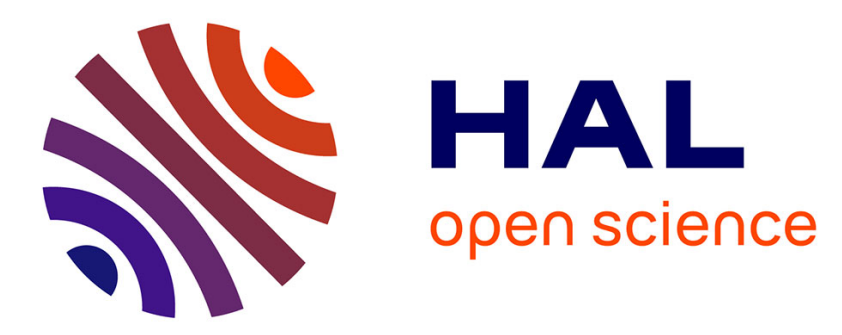

\title{
Semantic transportation planning for food products supply chain ecosystem within difficult geographic zones
}

Muhammad Ali Memon, Mohamed Hedi Karray, Agnès Letouzey, Bernard Archimède

\section{- To cite this version:}

Muhammad Ali Memon, Mohamed Hedi Karray, Agnès Letouzey, Bernard Archimède. Semantic transportation planning for food products supply chain ecosystem within difficult geographic zones. Industrial Management and Data Systems, 2017, vol. 117 (n09), pp. 2064-2084. 10.1108/IMDS-10201. hal-01977427

\section{HAL Id: hal-01977427 https://hal.science/hal-01977427}

Submitted on 10 Jan 2019

HAL is a multi-disciplinary open access archive for the deposit and dissemination of scientific research documents, whether they are published or not. The documents may come from teaching and research institutions in France or abroad, or from public or private research centers.
L'archive ouverte pluridisciplinaire HAL, est destinée au dépôt et à la diffusion de documents scientifiques de niveau recherche, publiés ou non, émanant des établissements d'enseignement et de recherche français ou étrangers, des laboratoires publics ou privés. 


\section{Open Archive Toulouse Archive Ouverte (OATAO)}

OATAO is an open access repository that collects the work of Toulouse researchers and makes it freely available over the web where possible.

This is an author-deposited version published in: http://oatao.univ-toulouse.fr/ Eprints ID: 19217

To link to this article:

DOI:10.1007/s10845-017-1344-0

URL: http://dx.doi.org/10.1007/s10845-017-1344-0

\section{To cite this version:}

Memon, Muhammad Ali and Karray, Mohamed Hedi and Letouzey, Agnès and Archimède, Bernard Semantic transportation planning for food products supply chain ecosystem within difficult geographic zones. (2017) Industrial Management \& Data Systems, vol. 117 (n09). pp. 2064-2084. ISSN 0263-5577 


\title{
Semantic transportation planning for food products supply chain ecosystem within difficult geographic zones
}

\author{
Muhammad Ali Memon \\ Institute of Information and Communication Technology, University of Sindh, \\ Jamshoro, Pakistan \\ Mohamed Hedi Karray and Agnès Letouzey \\ Laboratory Production Engineering, Ecole Nationale d'Ingenieurs de Tarbes, \\ Tarbes, France, and \\ Bernard Archimède \\ Ecole Nationale d'Ingenieurs de Tarbes, Tarbes, France
}

\begin{abstract}
Purpose - In difficult geographical zones (mountain, intra-cities areas, etc.), many shippers, from small and medium enterprises to individuals, may demand delivery of different food products (fresh, refrigerated, frozen, etc.) in small quantities. On the other side, carrier companies wish to use their vehicles optimally. Taking into account the perishability constraints (short-shelflife, temperature limits, etc.) of the transported food products and environmental constraints (pollution, carbon impact) while consolidating multiple kinds of food products to use vehicles optimally is not achieved by current transportation planning solutions. The purpose of this paper is to present an interoperable solution of a marketplace, formed by shippers and carriers, dedicated to the schedule of food transport orders.

Design/methodology/approach - This transportation planning system named Interoperable-Pathfinder, Order, Vehicle, Environment and Supervisor (I-POVES) is an interoperable multi-agent system, based on the SCEP (supervisor, customer, environment and producer) model (Archimede and Coudert, 2001). Ontologies are developed to create the planning marketplace comprising demands and offers from different sources (multiple shippers and carriers).

Findings - A hierarchy ontology for food products. A transporter system ontology. A global ontology that contains all shared concepts used by local ontologies of both shippers and carriers. I-POVES an interoperable model, which facilitates collaboration between carriers and their shippers through its active agents.

Practical implications - I-POVES is tested on a case study from the TECCAS Poctefa project, comprising transport and food companies from both sides of the Pyrenees (France and Spain).

Originality/value - There has been much work in the literature on the delivery of products, but very few on the delivery of food products. Work related to delivery of food products focuses mostly on timely delivery for avoiding its wastage. In this paper, constraints related to food products and to environment (pollution and carbon impact) of transport resources are taken into account while planning the delivery.
\end{abstract}

Keywords Ontology, Interoperability, Food perishability constraints, Multi-agents systems,

Transportation planning

Paper type Research paper

\section{Introduction}

In the transportation domain, distribution of large volumes is receiving greater attention (Ulku, 2012). Many efforts are being made for proposing solutions of transportation displacements concerning distribution to faraway locations in large quantities, and food 
products are not an exception (Hallman et al., 2015). Bringing food products from distinct regions across the countries and continents has become a regular routine in today's time. Consumers find products not grown or produced locally but brought in from distinct areas, making food business a global phenomenon.

Several solutions are developed and used efficiently by big distributor companies to bring food products in large quantities, using the capacity of large vehicles optimally (Chopra and Meindl, 2007). But when it comes to intra-cities transportation, or more generally geographically difficult zones, many small and medium enterprises located in these zones demand delivery in small quantities. Thus, food products are delivered in large quantities at the distribution centers located on the outskirts but close to these zones, from where they are transported in smaller quantities to the customers situated within those zones and vice versa. Big vehicles do not enter in these zones, they unload the products at the distribution center located at the beginning of city premises, from where products are delivered by small vehicles in small quantities (Caenegem et al., 2015). Such distribution is called as hub-and-spoke distribution (Bryan and O’Kelly, 1999).

The problem of using transport resources optimally in these zones becomes a crucial issue, because vehicle should not be loaded with less number of products than its full capacity (less than truckload (LTL) distribution) (Ulku, 2012; Hübner et al., 2016). Many small product delivery orders need to be shared (order sharing) or grouped for delivery (capacity sharing) in order to minimize the number of travelings, cost and especially environmental pollution (Yao and Song, 2013). This problem worsens when the transport orders concern perishable products.

However, in contrast to other products, food products, due to their perishability, bring more distribution constraints as weather conditions, tight schedule, short-shelflife, etc. (Wagner and Meyr, 2015). Due to that, food products can be categorized into different kinds like frozen, refrigerated, fresh, etc. with their respective constraints and different temperature requirements.

Therefore, transportation planning must take into account both food's perishability and vehicle's capacity with its temperature maintaining limits in order to consolidate orders. Transports are realized by specialized transport companies commonly known as third-party logistics (3PL) (Marasco, 2008; Mehmann et al., 2016) offering delivery services to their clients. These carriers may propose to deliver the order of same client, forming a competitive environment. They also require to collaborate with other carriers in order to deliver the products outside their limited operational territory.

In this context, both shippers and carriers together form a collaborative environment, which can be called a marketplace for transport services. Forming this marketplace involves a good understanding of information exchanged between shippers and carriers and between various carriers, especially about locations, food product constraints (short-shelflife), transport resource type (vehicle, train, plane), etc. Every entity in the marketplace has its own management system and standards. Hence, an interoperable mechanism is required to understand and transform heterogeneous information between these systems and achieve the collaboration.

This paper presents a solution of a transport marketplace to realize the schedules of transports for the delivery of food products achieved by the collaboration of heterogeneous shippers and carriers systems in difficult geographical zones.

In Section 2, the state of the art related to collaborative transportation planning is discussed. The adequacy of the SCEP (supervisor, customer, environment and producer) model for transportation is presented in Section 3. Section 4 details the proposed extension of the SCEP model named Interoperable-Pathfinder, Order, Vehicle, Environment and Supervisor (I-POVES). Section 5 is dedicated to the application of I-POVES on a case study 
extracted from the TECCAS project. Section 6 presents the limitations of the proposed approach and future perspective. Finally, this paper ends with conclusion including the future perspectives.

\section{Literature review}

Literature on collaborative transportation planning can be categorized into two main research areas: order sharing and capacity sharing (Verdonck et al., 2013). In order sharing, carriers combine and exchange delivery orders, while participating vehicles remain unchanged and they are located at specific locations (depots), where they start and terminate the delivery route. In capacity sharing, carriers tend to cooperate on sharing vehicle capacities. In contrast to order sharing, capacity sharing carriers maintains the confidentiality by not communicating the customer order information to other carriers. Carrier may reserve only capacity of other carriers and each carrier is solely responsible for its own order set. A detailed literature review on order sharing and capacity sharing is presented in the subsequent sections.

\subsection{Order sharing}

Order sharing can be further classified into two techniques: joint route planning and auction-based mechanisms (Verdonck et al., 2013).

2.1.1 Order sharing through joint route planning. In this technique, all customer orders are gathered in a central repository and efficient routing algorithms are applied to process those orders simultaneously (Cruijssen and Salomon, 2004; Cruijssen et al., 2007).

Cruijssen and Salomon (2004) considered the collaborative transportation network with multiple transporters and customers comprising a distribution center and many customer locations for delivery. This study focuses on comparing transportation costs of individual transporter with costs of the system in joint route planning formulated with the vehicle routing problem (VRP).

Similarly, Cruijssen et al. (2007) proposed the VRP with a time windows (VRPTW)-based framework to calculate the synergy value by joint route planning. In the considered transportation network, two nodes are connected by an arc with a certain Euclidian distance, where travel time considering these distance values is used to calculate the synergy values. An appropriate heuristic is constructed to minimize the number of routes. Another variant of VRP is multi-depot pickup and delivery problem (MDPDP) under time windows (MDPDPTW) as described in the VRP (Krajewska et al., 2008). MDPDP can be formulated as a directed Graph $\mathrm{G}=(\mathrm{V}, \mathrm{A})$, where $\mathrm{V}$ is the vertex set consisting of depots, pickup and delivery locations (D) and A is the arc set. To solve this problem, heuristic shifts from current solution to neighborhood solutions performing several removal and insertion operations to find out the vehicle routes, minimizing the total costs while respecting time windows. For the same MDPDPTW problem in a collaborative work comes the work of Dahl and Derigs (2011), who proposed sharing carriers in a dynamic environment for express customer orders.

On the contrary, to formulate the problem as a VRP, Liu et al. (2010) formulated the joint route planning as arc routing problems (ARP), a variant of VRPs in which vehicles are assigned to traverse certain arcs rather than certain nodes. Similarly a capacitated arc routing problem takes into consideration that order assigned to a vehicle for a route should not exceed its capacity (Golden and Wong, 1981).

2.1.2 Order sharing through auction-based mechanisms. In auction-based order sharing mechanisms, each carrier decides which customer requests need to be shared with other carriers in order to maximize the profit. 
Song and Regan (2004) proposed an auction-based request allocation mechanism based on combinatorial auctions. When a carrier receives a new customer order, it checks the profitability of the order to serve it by itself. If not, the carrier sets a reservation price and opens it for bidding from cooperating partners. The reservation price refers to the maximum value that the carrier is willing to pay to a partner carrier for the execution of the order. The other carriers then propose the bids with cost if they can execute it. Finally, the request originating carrier compares the bids and chooses the lowest bid if satisfactory.

Similarly, Figliozzi (2006) also proposed reservation prices to share the customer requests. However, his request assignment focuses on incentive compatible collaborative mechanisms (CMS). In CMS, collaborating carriers have to submit the delivery cost honestly corresponding to reservation cost in auction. It means that no carrier will pay more than its proposed reservation cost.

Krajewska and Kopfer (2006) proposed a three-steps-based allocation procedure using combinatorial auctions and game theory. In the first step, each carrier tries to calculate the cost of delivery by using its own available resources. In the second step, customer orders are exchanged in order to calculate the profit for entire collaborating partners. In the third step, matrix auction is created to identify the profitable delivery plans and sharing profits among the partners.

Similarly, Schwind et al. (2009) also proposed a combinatorial auction-based order exchange method in the context of the medium-sized logistics company, each operating in a certain region. As the delivery regions of these companies overlap, delivery costs can be reduced by sharing the orders.

Berger and Bierwirth (2010) also used combinatorial auction but are more concerned about information sharing. Here the collaboration is considered by means of centralized and decentralized manner for accounting the information sharing. In a centralized mean, all the customer requests are shared, while in a decentralized way, carrier chooses which requests to share and which not to share. They demonstrated that for information sharing, a decentralized method is superior for maintaining the confidentiality; however, a centralized method surely produces more profitable results.

Dai and Chen (2011) proposed order sharing on two decisions-based problem. The first decision is to choose requests that a carrier does not wish to serve and the second decision is to choose requests that are desirable to serve.

Wang and Kopfer (2014) proposed a combinatorial auction-based method for the allocation of orders, where participating logistics providers share complete vehicle routes instead of random group of single transport requests.

\subsection{Capacity sharing}

Instead of sharing customers' requests, carriers may share their vehicle's capacities through cooperation in order to better utilize their vehicle resources and maximize the profit. Agarwal and Ergun's (2010) work on capacity sharing problems for a fleet of ships can be treated in the same ways as trucks. Initially, the delivery routes are determined considering the customer request, then appropriate ships are allocated to those routes to improve capacity utilization. For optimal results, individual shipper company's ship is replaced by the combined fleet of collaborating shippers. Ship scheduling and route determination is formulated as a mixed-integer linear program.

Hern'andez et al. (2011) studied the capacity sharing problem as a dynamic LTL carrier collaboration for minimizing the route costs. They named this problem as a deterministic dynamic single carrier collaboration problem, because they take into consideration that each carrier uses its own capacity for the delivery before sharing it with other carriers. This problem is formulated as linear integer programming with an objective function and the goal is to minimize the total cost of the carrier. 
In contrast to minimizing the cost in capacity sharing problem, maximizing the profit instead is studied by Houghtalen et al. (2011). In this context, two approaches are proposed: limited control model and strict control model. In a limited control model, each individual carrier has limited control over other carriers as well as the limited use of vehicle capacity. In a strict control program, a single carrier has total control of other carrier's decisions as it can include other carrier's order as per its desire. In comparison, the limited control model seems to be feasible as the strict control model leads to inconsistencies.

Sprenger and Mönch (2011) studied the vehicle's capacity sharing at an intermediate distribution center in order to reduce delivery costs and use the loading capacity optimally. First, they decomposed the problem into VRP sub-problems. Second, they used greedy heuristic and ant colony optimization to solve those sub-problems.

A different approach on capacity sharing is presented by Fischer et al. (1996). They studied the capacity sharing in MARS environment. MARS stands for Modeling Autonomous Cooperating Shipping companies system, modeling the cooperative planning for logistics companies. The distinguish feature of this approach is that instead of companies scheduling the order, truck owners themselves take the decision. The decision is made through the process of auction and negotiation between the carriers offering the free space and other carriers to decide to accept the offer or not.

Reviewing both the collaboration techniques it can be deduced that through exchange of customer requests, carriers find an increase in the capacity usage, efficient resource management and reduction in total cost, while with capacity sharing, carriers find capital profit sharing and better rates of utilization of vehicles. Considering the literature, one of the future directions proposed is combining order sharing with capacity sharing. Dai and Chen (2012) suggested such approach for logistics collaboration in which both customer orders and vehicle capacities are combined together. In their work, freight forwarder serves as a decision maker based on the information collected for all the customer orders, vehicle capacities and transportation costs. They formulated the problem as a mixed-integer programing model.

Our approach also combines the characteristics of both order sharing and capacity sharing, which uses incremental auction-based mechanism for order assignment and construct the transportation network based on the ARP problem, where routes are already defined for vehicles with fixed capacities. Therefore, traditional planning operation research methods (heuristics, fuzzy logic, linear integer programming, etc.) are not applicable in this scenario. As an alternative to these approaches, several distributed planning approaches are developed with agent technology (Kalina et al., 2015) and most of the agent approaches are based on the Contract-Net protocol (Liang and Kang, 2016). Using the Contract-Net protocol, an announcement concerning a task to perform is sent within the multi-agent system. Bids are made by the agents capable of performing the task. The bids are collected and compared, and a contract is made with the agent which has made the best proposal. A well-known agent system is iCOMAS (cooperative multi-agent systems) (Sprenger and Mönch, 2011), whose framework proposes decomposing overall transportation problems into sub-problems and solve those sub-problems on an autonomous basis, but it lacks the determination of effect of decomposition on overall problems. Cooperation of humans and intelligent agents for auctioning in transportation logistics is implemented in a platform developed by CWI (National Research Center for Mathematics and Computer Science, Amsterdam, The Netherlands) and VOS logistics companies (Nijmegen, The Netherlands) (Robu et al., 2011). The Living Systems/Adaptive Transportation Networks system (Neagu et al., 2006) uses agent techniques (mostly constraint-reasoning type techniques) for dynamic transport optimization. The Magenta system (Skobelev et al., 2007) is another such system, which explores the use of swarm-based optimization techniques (Dorigo et al., 2008). The multi-agent based load consolidation system (Baykasoglu and Kaplanoglu, 2011) 
proposes grouping multiple orders together in a vehicle, but transport order agent is bound to accept the proposition from one truck agent. The limitation of the Contract-Net protocol in above systems is that an order is usually planned operation by operation without any competition with the other orders, which is one of the requirements of the transport marketplace. In a marketplace, customers need to compete with each other to get the best transport resource. Apart from these systems, the SCEP multi-agent system (Archimede and Coudert, 2001) insists on competition between customers as well as it institutes such marketplace for services. It proposes that the producer agents represented services of producers for the clients represented as customer agents. Customer agent demands the realization of a project, constituting a sequence of tasks to realize where each task précises the service to be realized. This mechanism between the agents is based on the auction mechanism. It is used in the context of production planning, where services are associated with the production activities. This is also used in the context of maintenance planning and cooperation of maintenance and production planning and has illustrated its generic nature. Therefore, the adequacy of the SCEP model for the considered transportation problem needs to be studied in order to find the limitations for the improvement (Figure 1).

\subsection{Adequacy of SCEP model for our transportation problem}

SCEP models the functioning of a marketplace, where services are negotiated between suppliers, providing these services, and customers, wishing to carry out projects requiring these services. To achieve that, suppliers must define the characteristics of services they offer in terms of aptitude degree, cost and quality, while customers have, for each project, to define their objective, in terms of expected time, cost and quality, as well as all the required services with their temporal and structural constrains needed to achieve them. SCEP has been used with success in the context of the distributed production (Archimede et al., 2014) and maintenance planning (Archimede and Coudert, 2001), where all required services of a project are known in advance (concept of routing) and associated with well-defined production services (turning, milling, etc.) and of systematic, conditional or curative maintenance services (cleaning, repair, oil changes, etc.), realized by (or on) not movable resources (machine, manufacturing cell, etc.).

In order to use this model for the considered transportation problem, a transport service can be defined as a non-stop elementary displacement of transport resources (truck, boat, etc.) between two locations. Therefore, it is impossible for customers to specify in advance for a transport order, the route, the mode of the transport and all the necessary services to be used. Furthermore, it is necessary to take into account the operational area of carriers, their specific

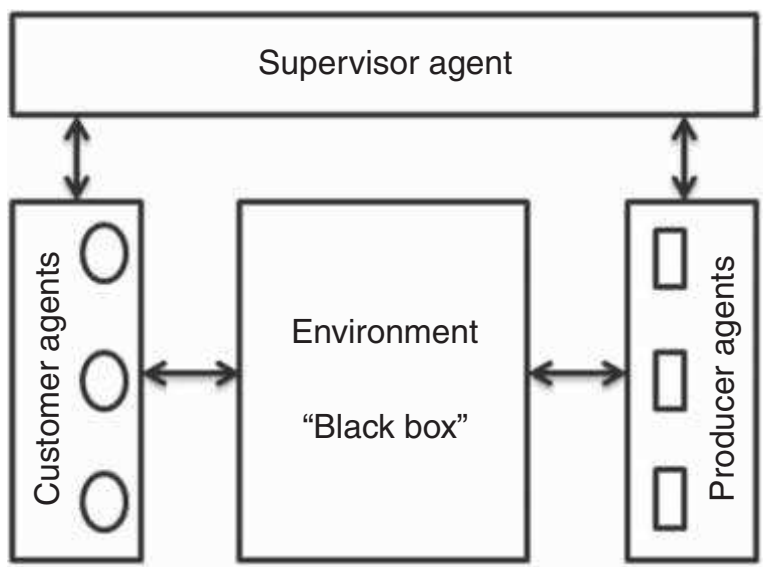

Source: Archimede and Coudert (2001)

Figure 1. SCEP model 
constraints for the mobility of transport resources and additional constraints induced by the transported entities including perishable food products, which are not handled in SCEP model. Reduction in cost and environmental impact leads to find the best possible mechanism of using transport capacity optimally through cooperation via transshipment hub between carriers operating on close geographic areas. In this distributed context, forecasting schedules require consideration of interoperability issues due to the heterogeneity of collaborating partners planning systems, which are not presently taken into account by the SCEP model.

By taking into account above limitations of SCEP for transportation issues (transport network, food product constraints, pollution and cost minimization and interoperability), it can be constituted that the SCEP model provides the basic structure of the marketplace but requires evolution to cope with above limitations. Therefore, authors evolve the SCEP model into a new generic model I-POVES which overcomes SCEP's limitations and is presented in next section.

\section{I-POVES multi-agent model}

I-POVES as shown in Figure 2 is composed of six basic components: supervisor agent, environment, virtual shipper (VS) agent, virtual carrier (VC) agent, pathfinder agent and a global ontology. An ontology provides a shared vocabulary (terminologies) and relationships, which can be used to model a knowledge domain (Gruber, 1993). In order to ensure interoperability, all agent components communicate with each other using a shared or (common) global ontology, modeling the knowledge domain of transportation and food product related concepts. Environment and supervisor agents have the same functioning as in the SCEP model. Environment is the blackboard for exchanging the information between active components. It simulates the ground for marketplace. Supervisor agent is responsible for managing the marketplace. It controls the access to Environment. Finally, pathfinder agent is responsible for determining the dynamic routing for transport orders.

The SCEP model establishes relationships in a marketplace between customer agents (each managing a single project) and producer agents (each managing one resource).

Figure 2. I-POVES architecture with shipper and carrier

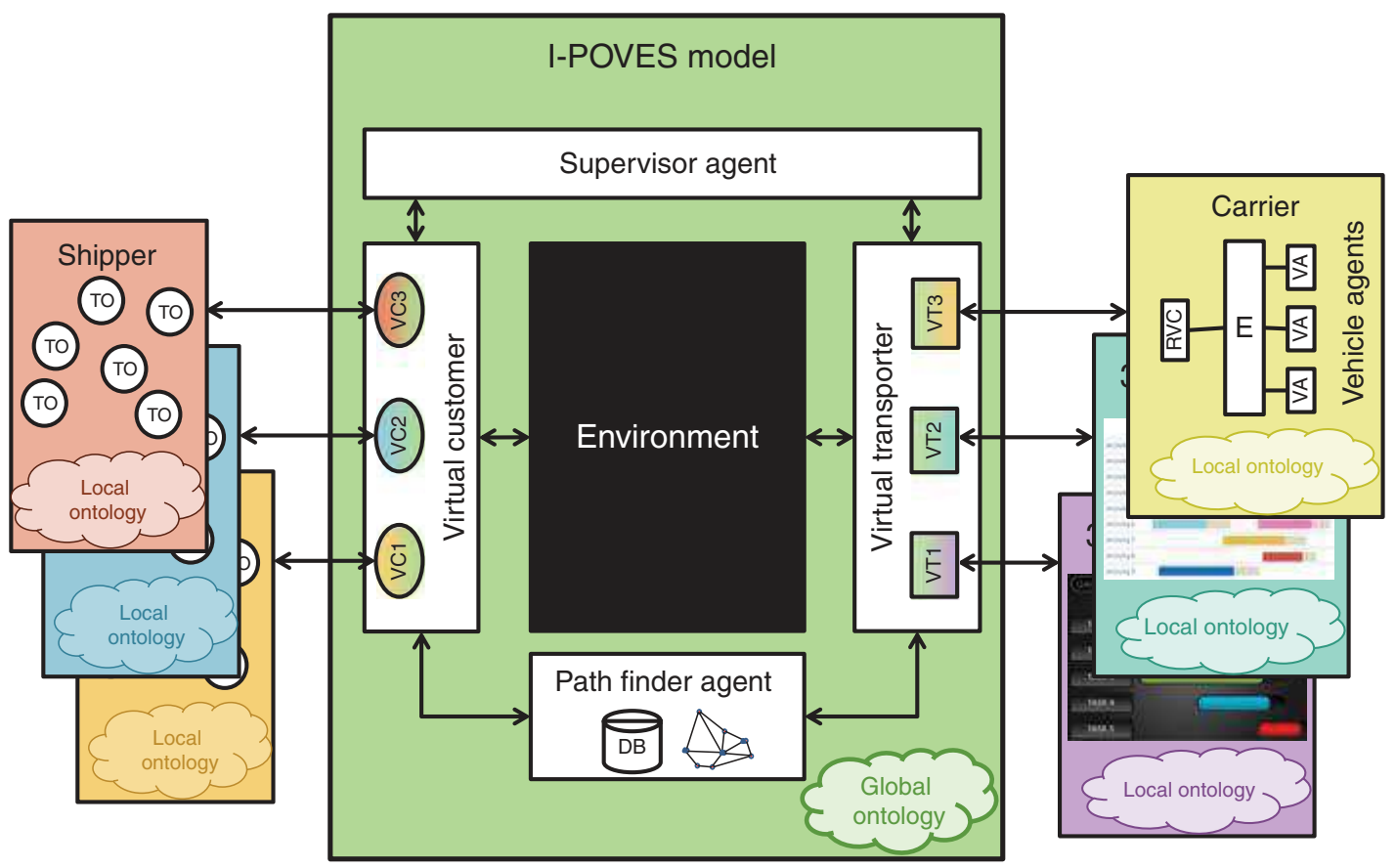


Similarly, I-POVES model establishes collaboration between VS agents (each manages transport orders of one shipper) and VC agents (each manages transport resources of one carrier enterprise). Shipper defines several transport orders using terms expressed into his own local product ontology. Similarly, a carrier operates in a limited geographical area, manages a fleet of vehicles and uses its own local ontology to describe vehicles characteristics and transportation network.

\subsection{Definition of transport services}

Unlike most of the production and maintenance resources, transport resources are cumulative, such as industrial ovens, meaning that their loading has to be constituted of compatible products. Additionally, defining an elementary displacement service as a non-stop travel between two locations is not sufficient to take into account existing incompatibilities between two types of products. It is, for example, inconceivable to transport goats and cabbage in the same compartment. In such kind of marketplace, the definition of transport services must take into account not only the origin and destination of the elementary displacement but also the characteristics of the transport resources. These characteristics should allow transport resources to easily identify products that can be loaded. This definition is based on a mapping mechanism between product and carrier ontologies.

Product ontology which is part of the shipper's system can be represented in the form of a hierarchy of categories (an example of a product ontology is shown in Figure 3). Top category is food (A0), which has further sub-categories (A1, A2, A3, etc.) and each sub-category has further sub-categories (A11, A21, A21, A31, A32, etc.). Finally, leaves in the hierarchy are instances, each representing a product. Each top to bottom category has its own characteristics; however, each category combines the characteristics of its sub-categories.

An example of carrier ontology is shown in Figure 4. It consists of the characteristics of transport resources. Any carrier wanting to adhere with I-POVES must model its ontology and VC dedicated for I-POVES. The VC defines transport services by integrating the product ontology with its local ontology.

A transport service is defined with origin, destination and product type, and have a syntax defined as "From $<$ Origin $>$ To $<$ Destination $>$ For $<$ ProductType $>$ ". Through mapping between previous carrier and product ontologies, following transport services are defined: FromOToBForA2, FromOToBForA22, FromOToBForA2, FromOToBForP1, FromBToCForA22, FromBToCForP1, FromCToDForA22, FromEToFForP1.

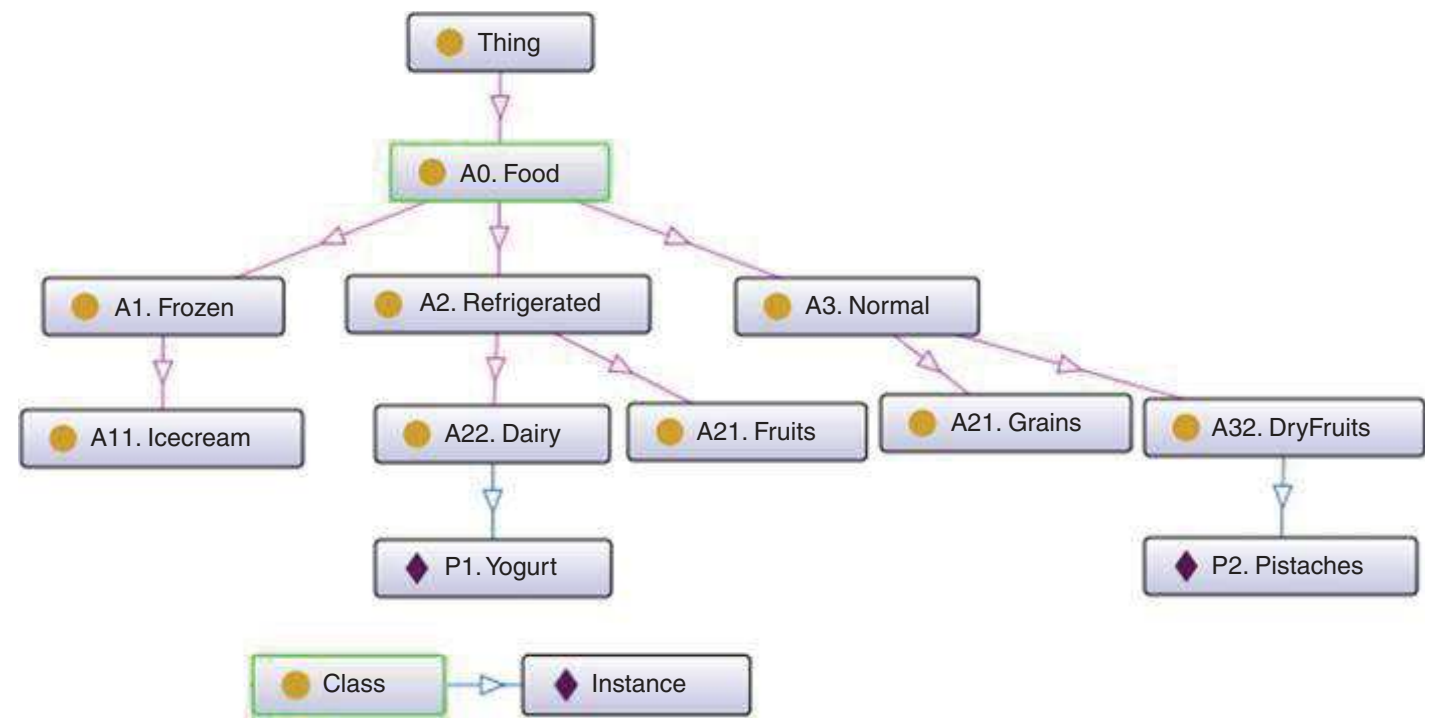

Figure 3.

Example of a product ontology 
Figure 4.

Example of a Carrier ontology

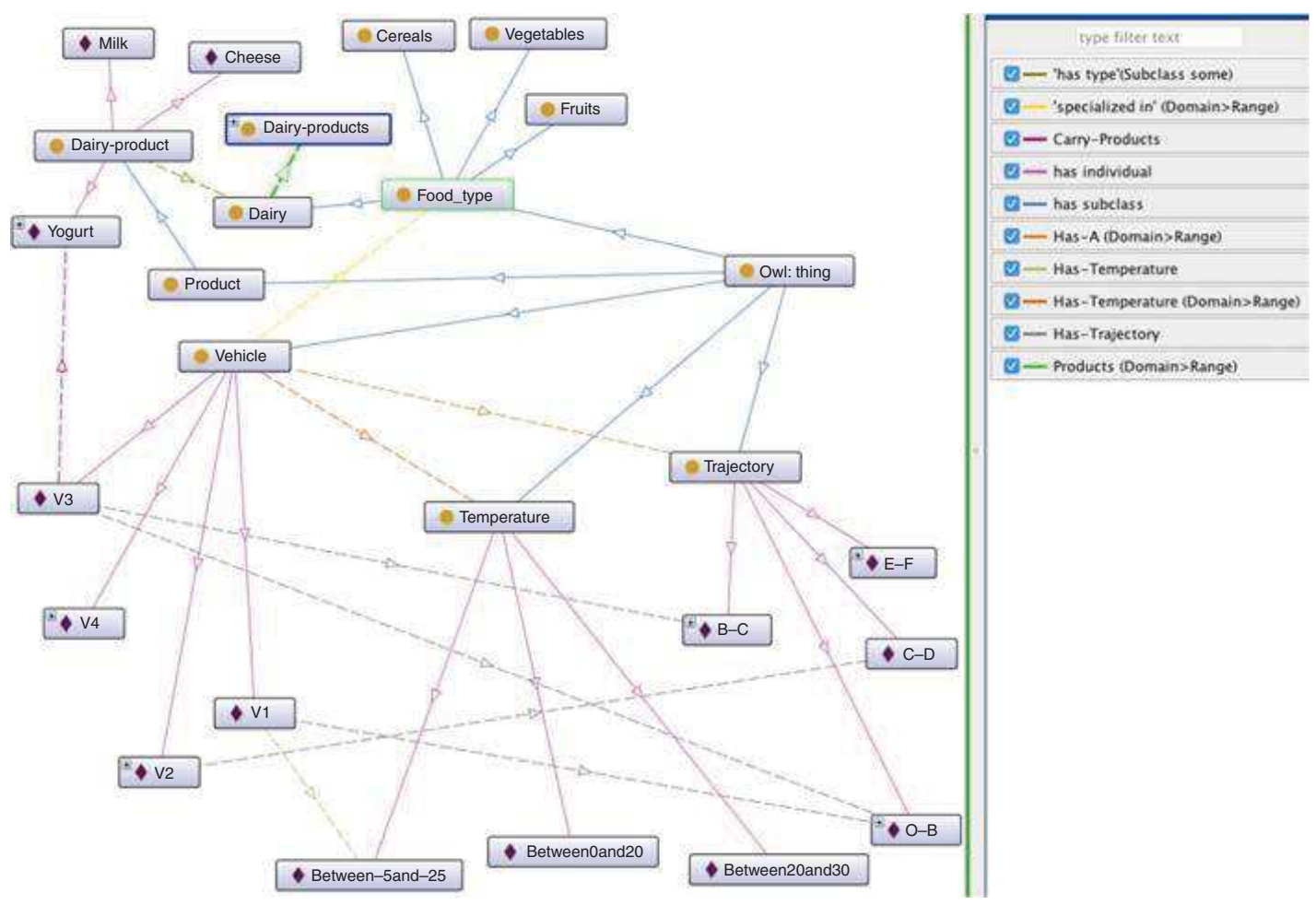

Alignment between these two ontologies is achieved in this work by using a semantic-based similarity algorithm proposed by Karray et al. (2010). This similarity algorithm applies three techniques for mapping, terminological, internal structure and extensional. In I-POVES, the authors used the terminological technique and implemented it using Semantic Web Rule Language (SWRL) (Horrocks et al., 2003) rules.

If a transport service is defined with a product category, then all its sub-categories can be consolidated. For example, for a category A2, products of type A21 and A22 can be consolidated. If a transporter resource cannot consolidate all the sub-categories of a category, then separate transport services for each product category (or a product itself) for that transport resource are defined. Product category ontology is incorporated in the global ontology residing in the core of I-POVES (shown later). Carrier ontology is explained in detail in Memon et al. (2014) and Memon (2014). Transport services of all the carriers adhering to I-POVES are sent through VC(s) to the pathfinder agent, to build the dynamic routing for transport orders. Based on that information, pathfinder constructs a complete network graph comprising of geographical locations associated with these transport services (Memon et al., 2014) from which the routing of each transport order is built.

\subsection{Collaboration method}

The collaboration method is based on the SCEP auction mechanism depicted in the study of Archimede and Coudert (2001). However, in order to best understand the following two sections, main characteristics of the auction will be recalled. The auction process contains several repeated cycles. However, only one cycle of auction is presented here.

The VS and VC agents get into negotiation mode. Each VS does the planning of all of its tasks (each task precise the transport service to be realized) optimistically: at the earliest with infinite capacity (without worrying about the actual vehicles capacities and availability). This planning gives, for each task, its wished position (WP), composed of required transport 
service, wished start date, wished end date and type of food product. For each task, concerned VC(s) propose two positions: an optimistic position (scheduled alone) named potential position (PP) and a more probable position (all tasks scheduled) named effective position (EP). $\mathrm{EP}$ (effective start date and effective end date) results from the scheduling with finite capacity of all the tasks collected by a VC. PP (potential start date and potential end date) results from the scheduling with finite capacity of only the considered task. At the end of this phase, each task owns a WP and several couples of PP and EP (one couple or each VC).

Afterwards, each VS starts the evaluation and validation process. The evaluation process is to seek the best effective and $\mathrm{PP}$ among the positions determined by $\mathrm{VC}$. That is to say, proposals that are most likely to reach the objective assigned to the VS for each transport order. Generally, a traditional planning system proposes only one start date and end date for each task (order). In that case, two position PP and EP are important to choose among all the positions received from carriers. The advantage of using PP is to help to determine whether the task should be validated (in validation process) for best EP or it is worth waiting for an improvement in proposals in the subsequent auction cycles.

\subsection{Interaction between shipper enterprise and VS}

Each shipper enterprise has its unique VS agent to connect with I-POVES. VS receives and manages transport orders from shippers in the form of their local ontology and translates them into the global ontology. For translation, VS comprises alignment of concepts between them on common semantics. After the planning of transport orders, VS translates these schedules from the global ontology to shipper's local ontology terminologies and sends schedules to the shipper enterprise. VS interacts with the shipper enterprise only in the start and end of the planning process.

\subsection{Interaction between carrier enterprise and VC}

Similar to shipper enterprise, each carrier enterprise has its own VC agent to connect with I-POVES. The VC agent matches and translates the concepts between carrier enterprise's local ontology and global ontology. If carrier's system adhering to I-POVES uses the same methodology for planning as I-POVES, functioning is more natural and notion of PP and $\mathrm{EP}$ is known by both. If carrier enterprise has a planning system, which proposes only final confirmed position after scheduling considering all the tasks, final positions correspond to EP of the I-POVES model. Therefore, in addition to ontology translations and EP collecting, VC should incorporate a mechanism for determining PPs from the carrier system. To collect PP, VC has to plan and undo the planning of transporter orders. If it is not possible, then it cannot be interoperated with I-POVES. Two solutions (a) and (b) can be adopted to assess PP.

In solution (a), $\mathrm{VC}$ sends a request of planning for one task at a time to the carrier system, sets planning result as PP for that task and then sends a request to the carrier system to undo that planning. This process is quite simple, but much time and resource consuming.

In solution (b), VC first forms different groups of tasks. Each group contains the tasks with the same origin, destination and type of food product and identical or nearby WP. Then, VC takes a single representative task from each group and forms a list of heterogeneous tasks, having different origin, destination, product type and WP. VC constructs several such lists until it covers all the groups. VC then sends the list to the carrier system, one task at a time for planning. When the carrier system finishes planning of one task, it sends dates to VC, VC sets PP of all the tasks of the corresponding group and sends request to carrier system to delete the current planning and built a new one with the next task. VC repeats the process for all the lists. Solution (b) is less time and resource consuming than (a), but it can deliver approximate results. 


\subsection{Global ontology}

Global ontology lies in the internal part of I-POVES. Figure 5 illustrates some portion of a global ontology. Global ontology contains two groups of concepts. One group is related to transportation planning algorithm concepts, while the other group is related to the concepts of food products and their constraints.

Transportation concepts used in the global ontology are loosely inspired from the ozone ontology developed by Becker and Smith (1998) and Smith et al. (2005). Our global ontology defines the concept "Vehicle," equipped with "Equipment." It includes concepts of "Transporter-order," "Objective," and "city." Geographical locations are represented by a chain of concepts like: "City," "Street," "Zone," etc. The class "Transportation-mode" has instances like "Train," "Road," "Sea," etc. Class "Transport-Order" is associated with class "Product," containing concepts related to food products.

Concepts of food products in the global ontology are inspired from the work described in the study by Kolchin and Zamula (2013) and Pizzuti and Mirabelli (2013). Class "Transport-Order" has object property "has-to-transport" with class "Product." Product has two sub-classes, "Food" and "Constraints." Class "Food" has further two sub-classes, "Food-Type," and "Food-Category." "Food-Type" represents different types of food products (bakery items, diary, etc.). The association between classes "FoodType" and "FoodCategory" is represented by the property "HasCategory," each instance of "FoodType" is associated with an instance of "FoodCategory." These instances are "High-Refrigerated," "Frozen" and so on. "Constraint" class contains the constraints of food represented by its instances "Short-ShelfLife," "Sunlight," "Humidity," and sub-class "Temperature." Another constraint called compatibility is handled in semantic rules as shown in Figure 6. The object property "CanTransport" between instances of class "Vehicle" and instances of class "FoodCategory." Indeed, we have defined as shown in Figure 6 SWRL rules, which allow each vehicle according to its resources the association of the products it can carry.

These are explained as follows:

- Temperature: it has six instances, each associated to a certain temperature limit represented by data property. For example, one instance is associated to $\left(T \min =-25^{\circ}, T \operatorname{Max}=+0^{\circ}\right)$ temperatures. Each instance of class "FoodCategory" is associated to one instance of "Temperature" class.

- Humidity: it is an instance of class "Constraint," associated with specific food products directly through object property "hasSensibilityWith." In our ontology, authors associate it with product instances like "butter" and "ice cream" of class "DairyFood."

- Sunlight: it is an instance of class "Constraint," associated with all the food products directly, except the food type of live animals, through object property normally.

- Short-ShelfLife: it is an instance of class "Constraint," associated with the food types of "FruitandVegetable," "MeatProduct," and "SeaFood" through object property.

- Compatibility: this constraint is handled by object property "hasNoCompatibilityWith." It is associated with different food categories, for example, category "Frozen" has no compatibility with "RoomTemperature" category. If a transport carrier is carrying products of "DeepFrozen" category, it cannot group with products of "RoomTemperature" type or vice versa.

Global ontology provides the federation of concepts of shipper and carrier ontologies. Local ontologies are subjected to evolve. This evolution will cause the enrichment of these local ontologies, also forcing the enrichment of global ontology at the same time in order to 


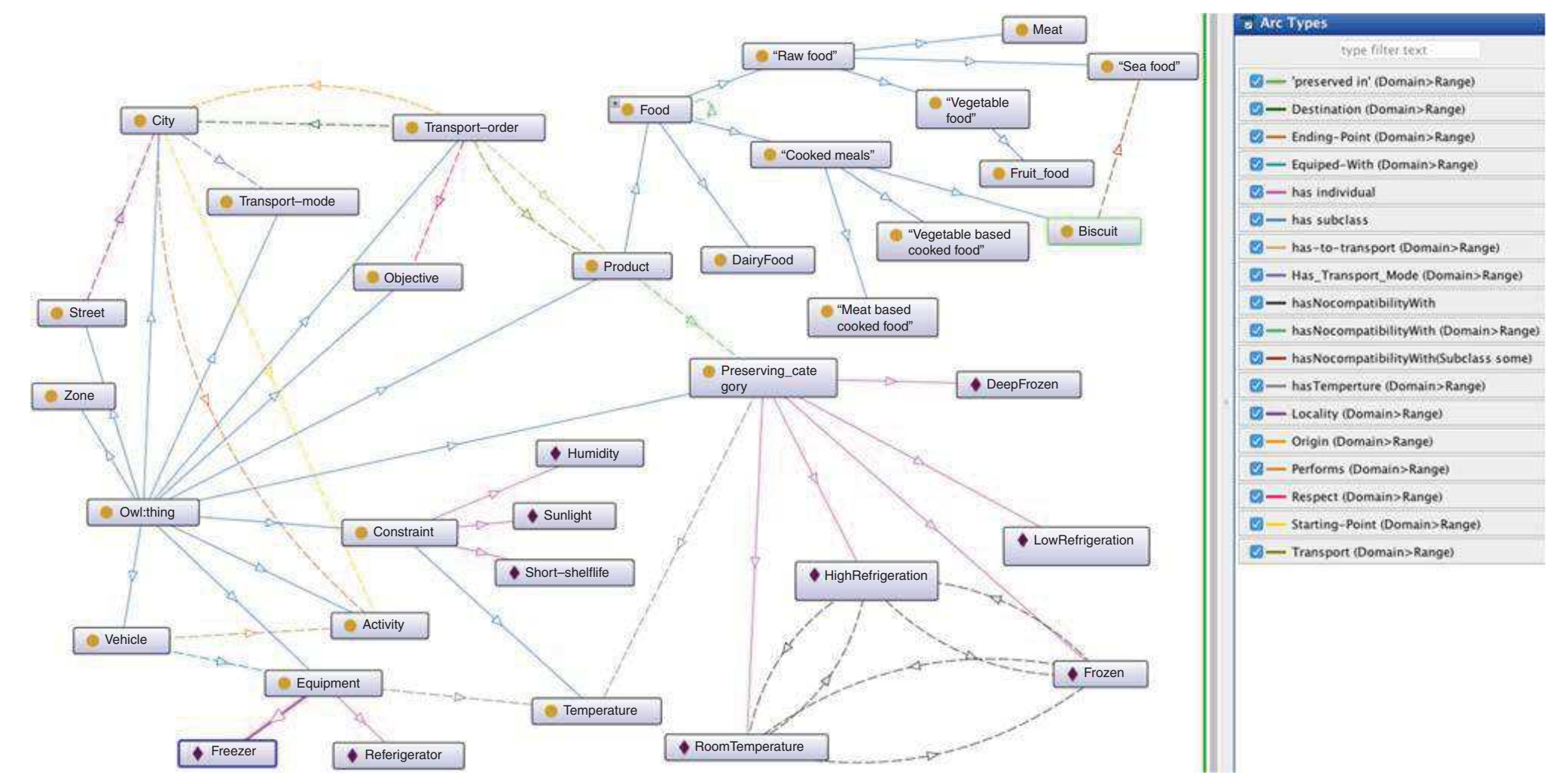


keep the compliance. The use of local and global ontologies provides liberty to shippers and carriers to work on their own standards without bothering everybody else's. Example of local ontologies of shipper and customer system are given in the study by Memon et al. (2014).

\subsection{Global functioning of I-POVES}

Global functioning of I-POVES is elaborated in eight steps as follows:

- Step 1: interoperating with carrier: VC receives the transporter vehicle and network information from carrier system in the format of its local ontology.

- Step 2: transformation of transport information into global ontology: VC transforms carrier information into global ontology and then sends it to the pathfinder agent, which updates its local database with that information. It constructs a large transport network combining the different zones operated by carriers.

- Step 3: interoperating with shipper: VS receives the transport orders from shipper in the form of its local ontology.

- Step 4: transformation of transport orders into global ontology: VS transforms transport orders into the format of global ontology based on the alignment embedded in it.

- Step 5: finding the route: all VS agents are invited by the supervisor agent to contact the Pathfinder agent in order to obtain possible traveling routes for transport orders. Based on the transportation network graph, Pathfinder agent elaborates for each managed transport order the traveling route (routing). For order, these are the sequence of tasks (elementary non-stop displacements), where each task corresponds to a transport service achieved by transporter vehicle(s).

- Step 6: (scheduling phase 1) start of collaboration process: process of negotiation between VS and VC through the environment starts with the determination of WP by VS.

- Step 7: (scheduling phase 2) vehicle agents proposals: in response to the auction, $\mathrm{VC}$ determines PP and EP.

- Step 8: (scheduling phase 3) evaluation and validation of the positions: afterwards, each VS comes and reads the positions in the environment and starts the evaluation and validation process as explained in the collaborative process (Archimede and Coudert, 2001).

\section{Application}

Case study considered here is shown in Figure 7 and has been extracted from the European project TECCAS. TECCAS name is derived from the Spanish words "Desarrollo de TEcnologias orientadas a favorecer la Colaboración entre agentes de la Cadena Alimentaria de Suministro," meaning that its objective was the "Development of technologies to promote collaboration between the actors of food supply chain" in the border area of France and Spain.

Figure 6. Rules for compatibility constraint

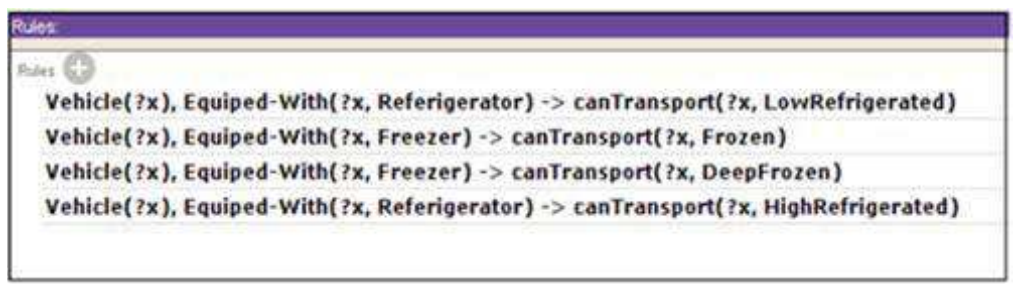




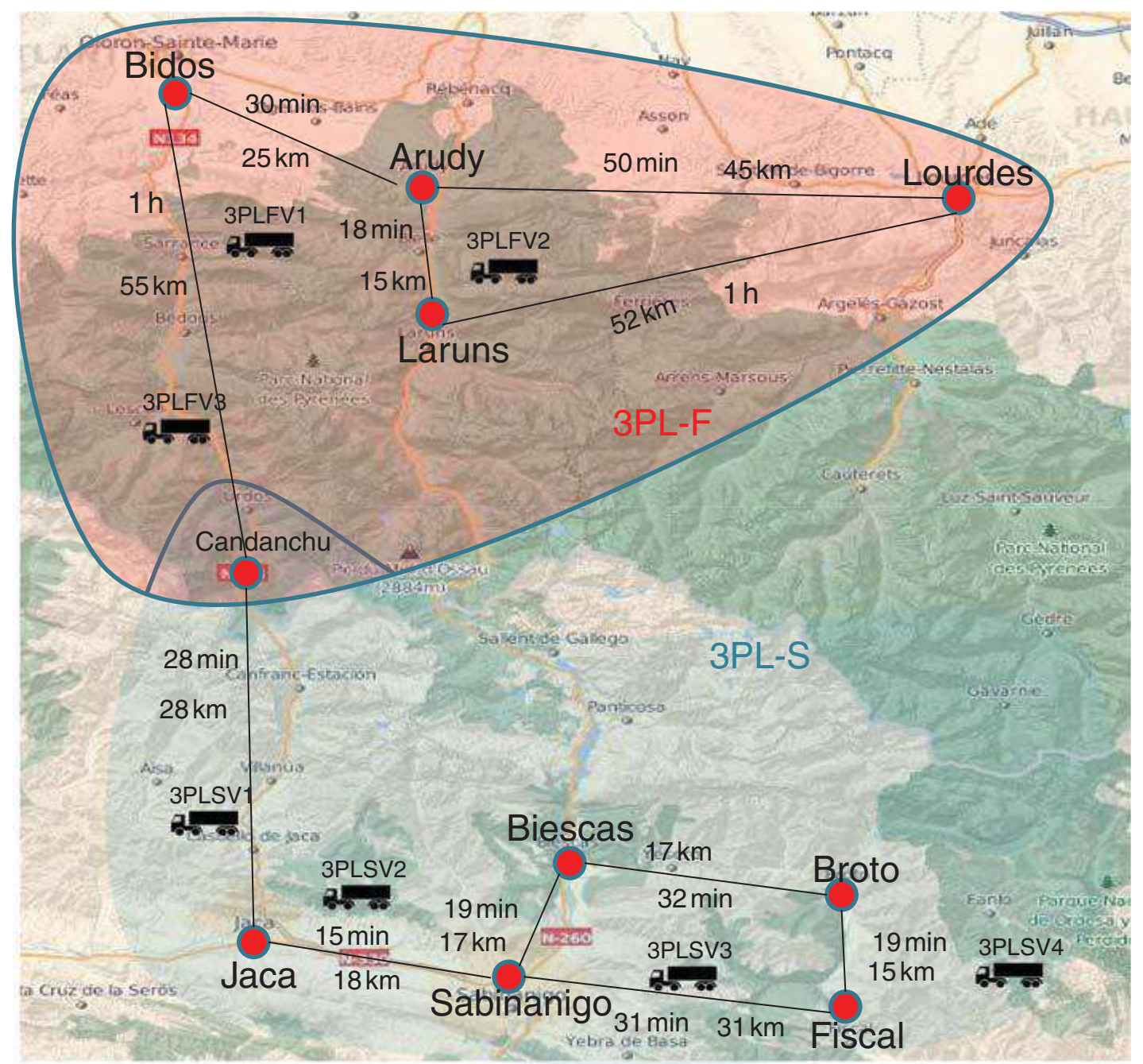

Figure 7. Case study from TECCAS project

Its duration was two years from 2012 to 2014 and it had four partners two from industry and two from research. Case study comprises carriers and shippers operating in the cross-border area of France and Spain, a mountain area, geographically difficult. It contains shipper companies, one situated in Spain (Shipper-S) and other situated in France (Shipper-F), and similarly two carriers. One carrier operating in the south of France is called 3PL-F. Other carrier operates in the north area of Spain and is called 3PL-S. 3PL-F and 3PL-S share an intermediate distribution center at Candanchu city for loading and unloading goods between France and Spain. Both carriers are specialized in transporting food products and manage their own fleet of vehicles equipped to maintain certain temperature for different food products. 3PL-F owns three vehicles and 3PL-S owns four vehicles. In this case study, it is assumed that all the vehicles have fixed itineraries and fixed pre-calculated timetables of departure for each elementary non-stop displacement associated with them.

The implementation of this case study is based on the three instances of I-POVES. One instance is for collaborative purpose and two instances are for local planner for 3PL-F and 3PL-S. Collaborative I-POVES receives three transport orders from Shipper-F as well as Shipper-S, through VS(F) and VS(S), respectively, and transforms them into global ontology.

$\mathrm{VC}(\mathrm{F})$ and $\mathrm{VC}(\mathrm{S})$, after receiving concerned information from 3PL-F and 3PL-S, respectively, based on their alignment, generate elementary transportation services for each non-stop elementary displacements. These transport services are then sent to pathfinder 
Table I.

Transport services stored in I-POVES database agent to construct a transportation network graph. The pathfinder agent associates a unique "identifier" to each transport service (see Table I).

Based on these transportation network information, for all the transport orders, the pathfinder agent determines the routing (example is shown for TO3 and TO4 in Table II).

In this paper, details of the different cycles of auction in collaborative method are not explained as they are already explained in detail for core SCEP in the study by Archimede and Coudert (2001). Figure 8 illustrates the planning results generated by 3PL-F local

\begin{tabular}{llcl}
\hline Identifier & Transport service & Identifier & Transport services \\
\hline 1 & FromBidosToArudyforRefrigerated & 2 & FromArudyToBidosRefregerated \\
3 & FromArudyToLourdesforFrozen & 4 & FromLourdesToArudyfor Frozen \\
5 & FromLarunsToArudyforRefrigerated & 6 & FromArudyToLarunsforRefrigerated \\
7 & - & 8 & - \\
9 & - & 10 & -
\end{tabular}

Table II.

Routing determined by Pathfinder

\begin{tabular}{lclc}
\hline Transport order & Route & Transport service & Duration \\
\hline \multirow{2}{*}{ TO3 } & 1 & FromBidosToArudyforReferigerated & $30 \mathrm{~min}$ \\
& & FromArudyToLarunsforRefrigerated & $18 \mathrm{~min}$ \\
TO4 & 2 & FromBiescasToSabinanigoRefrigerated & $19 \mathrm{~min}$ \\
& & - & $15 \mathrm{~min}$ \\
& & & - \\
& & & - \\
& & &
\end{tabular}

Figure 8.

Planning generated by 3PL-F I-POVES

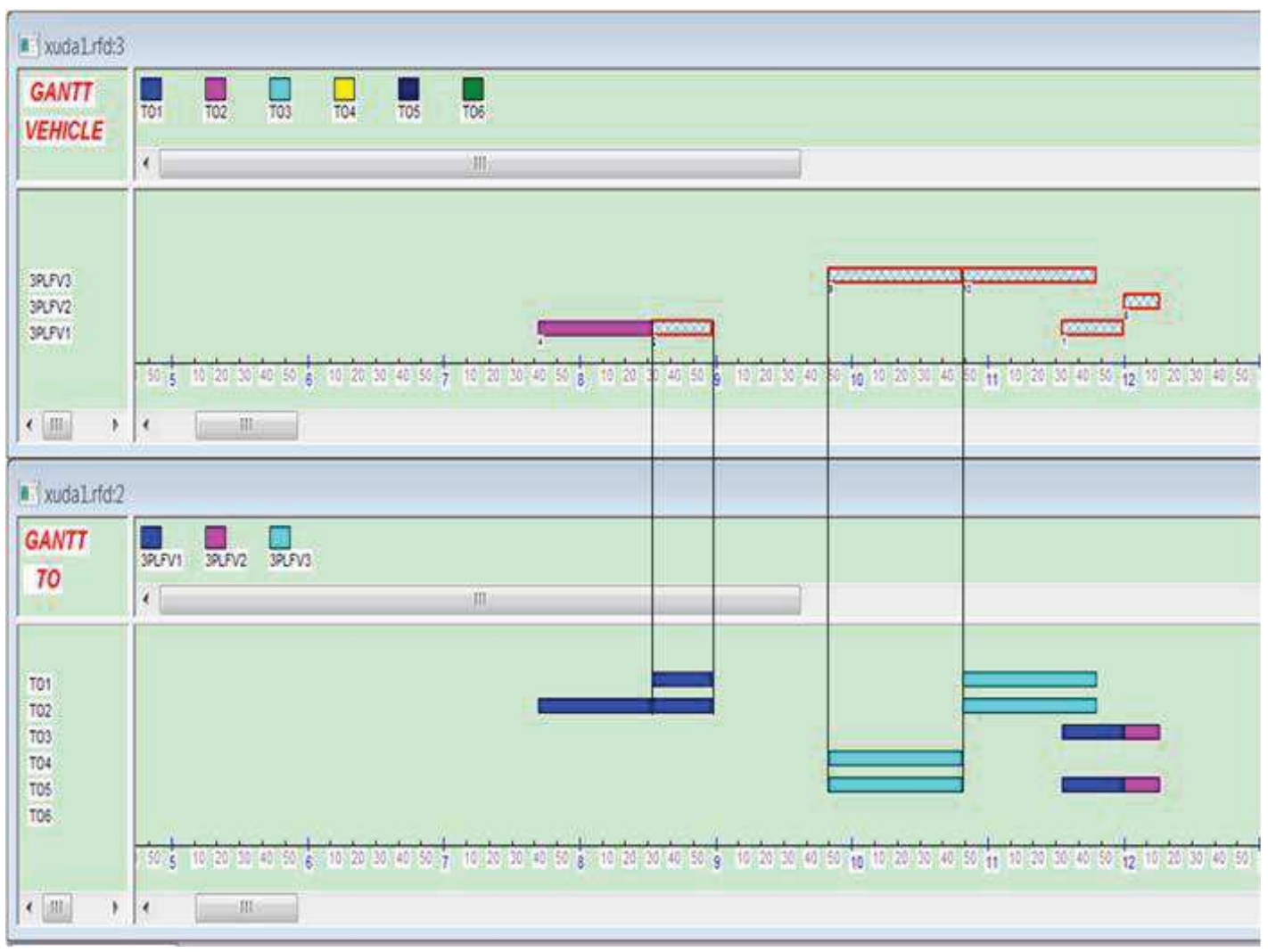


planner in the form of Gantt charts: from order perspective (GANTT TO window, top of Figure 8), from vehicle perspective and service perspective (GANTT VEHICLE window, bottom of Figure 8).

GANTT TO window contains a list of transport orders on the vertical axis, the time on the horizontal axis and their corresponding scheduled tasks inside the chart. On top of the GANTT TO, there is list of vehicles represented by a unique color. Same color of the task and vehicle indicates which vehicle is transporting this task. GANTT VEHICLE window contains a list of vehicles of 3PL-F on the vertical axis, the time on the horizontal axis and tasks inside the chart. On top of the GANTT VEHICLE window, list of transport orders are represented by a unique color. Same color of task and transport order represents that this vehicle is executing the task for that particular transport order. All the colored tasks in GANTT VEHICLE show individual tasks. Tasks with red boundary and sky blue crosses represent consolidated tasks. In I-POVES, a simple consolidation criteria is implemented based on order timing and vehicle capacity. After matching the product category, if a transport order's WP coincides with other for vehicle's departure and if vehicle has enough capacity to carry both, then I-POVES consolidates both orders. 3PL-F plans total of five tasks consolidation of order 1 and 2 (both belong to Frozen category), orders 4 and 5 (Refrigerated category) and orders 3 and 5 (Refrigerated category).

Figure 9 illustrates the planning results generated by 3PL-S local planner. 3PL-S proposes total of three tasks consolidation of order 1 and 2 (Frozen category).

Finally, Figure 10 represents the collaborative planning of both 3PL-F and 3PL-S together for the delivery of all six transport orders generated by collaborative I-POVES.

Objective of this case study is to show the functioning of I-POVES in a simplified manner. It will be further tested with much larger data sets in order to illustrate its efficiency. As this approach is different from all the approaches found in the literature, authors cannot clearly compare this approach with other collaborative transportation planning approaches.

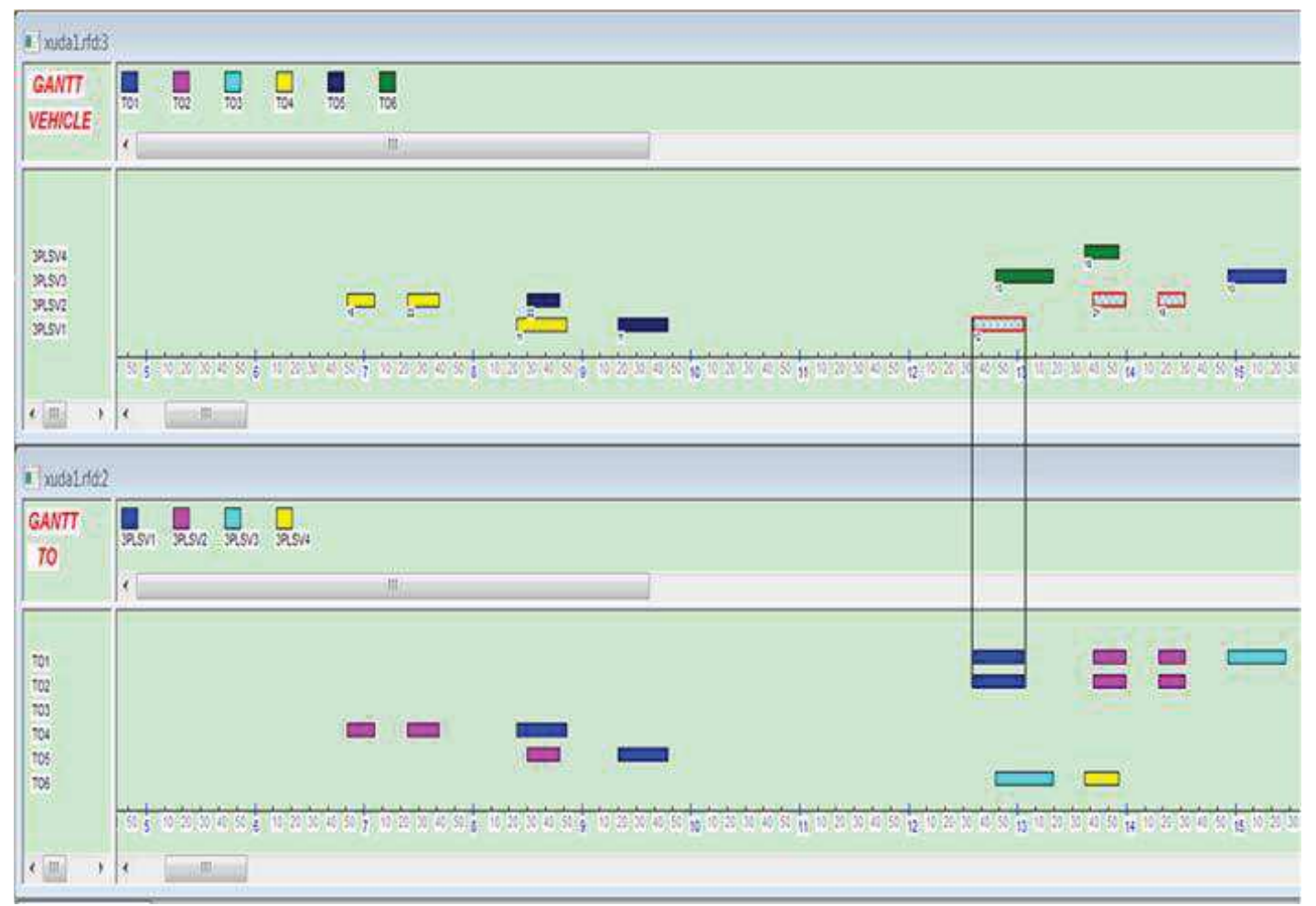

Figure 9. 


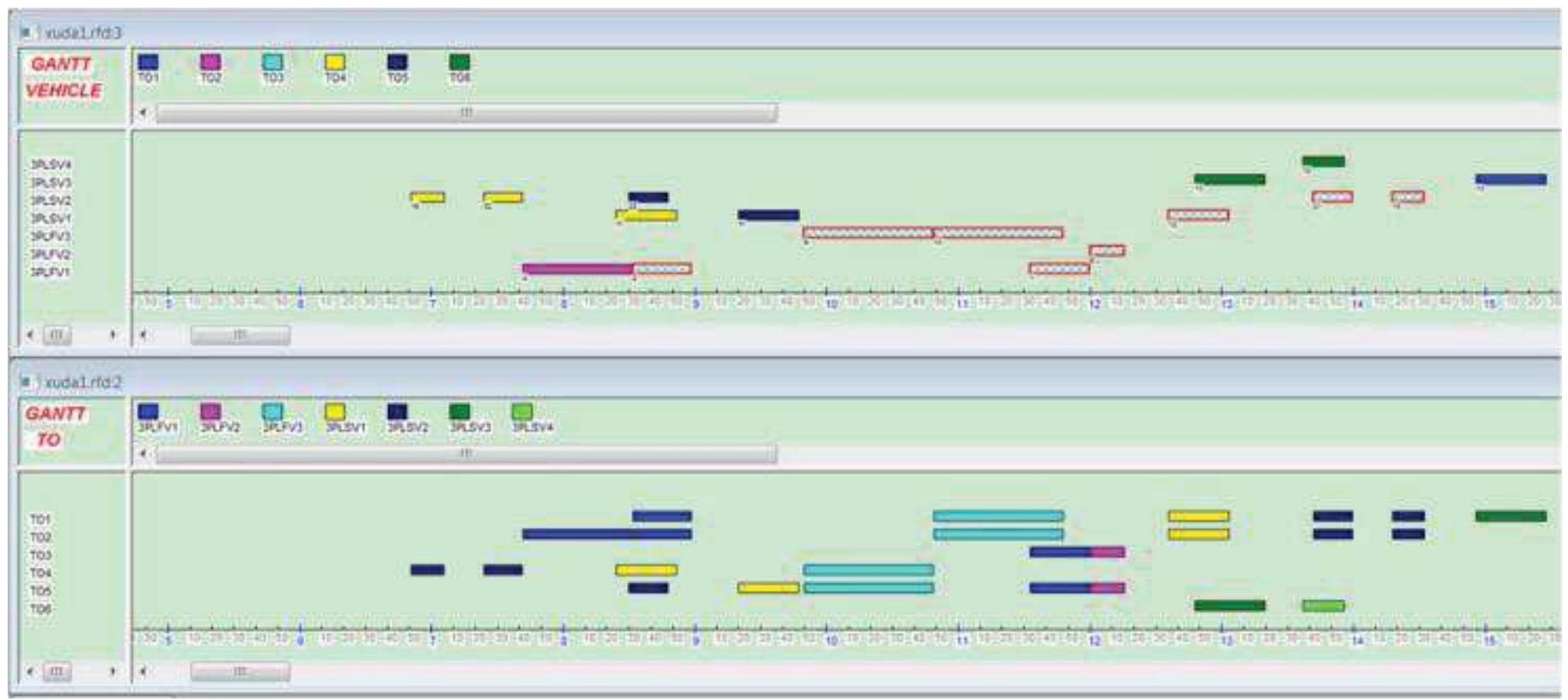




\section{Limitations and perspective}

I-POVES does not take into account at this stage perturbation during transportation and reactive strategies to handle them; however, reactive strategies are implemented in its ancestor model SCEP. They just need to be adapted in I-POVES. I-POVES does not consider the different cost structures of different type of products: frozen, refrigerated, normal, etc. I-POVES does not have any mechanism to divide the transport order product quantity among multiple vehicles, in case if one vehicle lacks the capacity to transport it completely.

Future directions include first and immediate perspective to implement work on limitations presented above. Second, to study state of the art of tracing and tracking methods and approaches and consider the food security information such as origin, lot, manufacturing date, best before date, etc. in order to improve planning. Third, researching price negotiation mechanism and consider product weight and size parameters. Finally, testing the I-POVES model with a large data set and improve the GUI visualization of the scheduling results.

\section{Conclusion}

In order to deliver the food products in small quantities in difficult zones or inside cities, a model I-POVES is proposed, which simulates a marketplace for offering and acquiring transport services. I-POVES is an extension of the generic SCEP model. I-POVES's functionality, however, is not limited into these difficult zones, but its generic nature elevates its utilization in case of transportation planning of large volumes and faraway locations. I-POVES facilitates collaboration between carriers and their shippers through its active agents. I-POVES is embedded with a global ontology that contains all shared concepts used by local ontologies of both shippers and carriers. Shippers demand delivery of food products, where each product is associated with certain perishability constraints. Carriers on the other hand possess transport resources, which have their own characteristics to load different food products. I-POVES planning mechanism handles the correspondences between these transport resources characteristics and products perishability constraints in order to facilitate the consolidation of transport orders and fill up the transport resource capacity at maximum, reduce the number of travels. The reduction in number of transport travels not only yield the reduction of transportation cost but also the reduction in $\mathrm{CO} 2$ emission. The reduction in $\mathrm{CO} 2$ emission will lead to a decrease in the environmental pollution and will cause positive effect on people's health. A reduction in the transport cost will cause a reduction in product prices that will make global impact of reduction on overall inflation rate. Future directions for our work include studying the load consolidation mechanisms at the planning level, introducing tracking and tracking methods, considering product size, weight and volume particulars, etc.

\section{References}

Agarwal, R. and Ergun, O. (2010), "Network design and allocation mechanisms for carrier alliances in liner shipping", Operations Research, Vol. 58 No. 6, pp. 1726-1742.

Archimede, B. and Coudert, T. (2001), "Reactive scheduling using a multi-agent model: the SCEP framework", Engineering Applications of Artificial Intelligence, Vol. 14 No. 5, pp. 667-683.

Archimede, B., Letouzey, A., Memon, M.A. and Xu, J. (2014), "Towards a distributed multi-agent framework for shared resources scheduling”, Journal of Intelligent Manufacturing, Vol. 25 No. 5, pp. 1077-1087.

Baykasoglu, A. and Kaplanoglu, V. (2011), "A multi-agent approach to load consolidation in transportation”, Advances in Engineering Software, Vol. 42 No. 7, pp. 477-490. 
Becker, M. and Smith, S.F. (1998), An Ontology for Multi-Modal Transportation Planning and Scheduling, The Robotics Institute, Carnegie Mellon University, Pittsburgh, PA.

Berger, S. and Bierwirth, C. (2010), "Solutions to the request reassignment problem in collaborative carrier networks", Transportation Research Part E: Logistics and Transportation Review, Vol. 46 No. 5, pp. 627-638.

Bryan, D.L. and O'Kelly, M.E. (1999), "Hub-and-spoke networks in air transportation: an analytical review", Journal of Regional Science, Vol. 39 No. 2, pp. 275-295.

Caenegem, W.V., Drahos, P. and Cleary, J. (2015), "Provenance of Australian food products: is there a place for geographical indications", Rural Industries Research and Development Corporation, Vol. 15 No. 60, pp. 15-60.

Chopra, S. and Meindl, P. (2007), Supply Chain Management. Strategy, Planning \& Operation, Springer, Prentice Hall, Upper Saddle River, NJ.

Cruijssen, F. and Salomon, M. (2004), "Empirical study: order sharing between transportation companies may result in cost reductions between 5 to 15 percent", CentER Discussion Paper No. 2004-80.

Cruijssen, F., Bräysy, O., Dullaert, W., Fleuren, H. and Salomon, M. (2007), "Joint route planning under varying market conditions", International Journal of Physical Distribution \& Logistics Management, Vol. 37 No. 4, pp. 287-304.

Dahl, S. and Derigs, U. (2011), "Cooperative planning in express carrier networks - an empirical study on the effectiveness of a real-time decision support system", Decision Support Systems, Vol. 51 No. 3, pp. 620-626.

Dai, B. and Chen, H. (2011), "A multi-agent and auction-based framework and approach for carrier collaboration”, Logistics Research, Vol. 3 Nos 2-3, pp. 101-120.

Dai, B. and Chen, H. (2012), "Mathematical model and solution approach for carriers' collaborative transportation planning in less than truckload transportation", International Journal of Advanced Operations Management, Vol. 4 Nos 1-2, pp. 62-84.

Dorigo, M., Birattari, M., Blum, C., Clerc, M., Stützle, T. and Winfield, A. (Eds) (2008), “Ant colony optimization and swarm intelligence", Proceedings of 6th International Conference, ANTS 2008, Vol. 5217, Springer, Brussels, September 22-24.

Figliozzi, M.A. (2006), "Analysis and evaluation of incentive compatible dynamic mechanisms for carrier collaboration", Transportation Research Record: Journal of the Transportation Research Board No. 1966, Transportation Research Board of the National Academies, Washington, DC, pp. 34-40.

Fischer, K.P.J.R. and Pischel, M. (1996), "Cooperative transportation scheduling: an application domain for DAI”, Applied Artificial Intelligence, Vol. 10 No. 1, pp. 1-34.

Golden, B.L. and Wong, R.T. (1981), "Capacitated arc routing problems", Networks, Vol. 11 No. 3, pp. 305-315.

Gruber, T.R. (1993), "A translation approach to portable ontology specifications", Knowledge Acquisition, Vol. 5 No. 2, pp. 199-220.

Hallman, W.K., Senger-Mersich, A. and Godwin, S.L. (2015), "Online purveyors of raw meat, poultry, and seafood products: delivery policies and available consumer food safety information", Food Protection Trends, Vol. 35 No. 2, pp. 80-88.

Hern'andez, S., Peeta, S. and Kalafatas, G. (2011), "A less-than-truckload carrier collaboration planning problem under dynamic capacities", Transportation Research Part E: Logistics and Transportation Review, Vol. 47 No. 6, pp. 933-946.

Horrocks, I., Patel-Schneider, P.F., Boley, H., Tabet, S., Grosof, B. and Dean, M. (2003), "SWRL: semantic web rule language combining OWL and RuleML", Version 0.6, DAML, available at: www.daml. org/2003/11/swrl

Houghtalen, L., Ergun, O. and Sokol, J. (2011), "Designing mechanisms for the management of carrier alliances", Transportation Science, Vol. 45 No. 4, pp. 465-482. 
Hübner, A., Kuhn, H. and Wollenburg, J. (2016), "Last mile fulfilment and distribution in omni-channel grocery retailing: a strategic planning framework”, International Journal of Retail \& Distribution Management, Vol. 44 No. 3, pp. 228-247.

Kalina, P., Vokrinek, J. and Marik, V. (2015), "Agents toward vehicle routing problem with time windows", Journal of Intelligent Transportation Systems, Vol. 19 No. 1, pp. 3-17.

Karray, M.-H., Chebel-Morello, B. and Zerhouni, N. (2010), "A contextual semantic mediator for a distributed cooperative maintenance platform”, Osaka University Nakanoshima Center, Osaka, pp. 181-188.

Kolchin, M. and Zamula, D. (2013), "Food product ontology: initial implementation of a vocabulary for describing food products", Proceeding of the 14th Conference of Open Innovations Association FRUCT, Helsinki, November, pp. 11-15.

Krajewska, M.A. and Kopfer, H. (2006), "Collaborating freight forwarding enterprises”, OR Spectrum, Vol. 28 No. 3, pp. 301-317.

Krajewska, M.A., Kopfer, H., Laporte, G., Ropke, S. and Zaccour, G. (2008), "Horizontal cooperation among freight carriers: request allocation and profit sharing", Journal of the Operational Research Society, Vol. 59 No. 11, pp. 1483-1491.

Liang, H. and Kang, F. (2016), "A novel task optimal allocation approach based on contract net protocol for agent-oriented UUV swarm system modeling", Optik-International Journal for Light and Electron Optics, Vol. 127 No. 8, pp. 3928-3933.

Liu, R., Jiang, Z., Fung, R.Y., Chen, F. and Liu, X. (2010), "Two-phase heuristic algorithms for full truckloads multi-depot capacitated vehicle routing problem in carrier collaboration", Computers \& Operations Research, Vol. 37 No. 5, pp. 950-959.

Marasco, A. (2008), "Third-party logistics: a literature review", International Journal of Production Economics, Vol. 113 No. 1, pp. 127-147.

Mehmann, J., Teuteberg, F. and Al-Mashari, M. (2016), "Process reengineering by using the 4PL approach - a case study on transportation processing in the agricultural bulk logistics sector", Business Process Management Journal, Vol. 22 No. 4, pp. 879-902.

Memon, M.A. (2014), "Transportation interoperable planning in the context of food supply chain", doctoral dissertation, Ecole Doctorale Systèmes, Toulouse.

Memon, M.A., Letouzey, A., Karray, M.H. and Archim'ede, B. (2014), "Collaborating multiple 3PL enterprises for ontology-based interoperable transportation planning", Enterprise Interoperability VI: Interoperability for Agility, Resilience and Plasticity of Collaborations, Vol. 7, p. 319.

Neagu, N., Dorer, K., Greenwood, D. and Calisti, M. (2006), "LS/ATN: reporting on a successful agentbased solution for transport logistics optimization", IEEE workshop on Distributed Intelligent Systems: Collective Intelligence and its Applications, IEEE, pp. 213-218.

Pizzuti, T. and Mirabelli, G. (2013), "Ftto: an example of food ontology for traceability purpose", IEEE 7th International Conference on Intelligent Data Acquisition and Advanced Computing Systems (IDAACS), Vol. 1, September, pp. 281-286.

Robu, V., Noot, H., Poutré, H.L. and Schijndel, W.-J.V. (2011), "A multi-agent platform for auction-based allocation of loads in transportation logistics", Expert Systems with Applications, Vol. 38 No. 4, pp. 3483-3491.

Schwind, M., Gujo, O. and Vykoukal, J. (2009), "A combinatorial intra-enterprise exchange for logistics services”, Information Systems and e-Business Management, Vol. 7 No. 4, pp. 447-471.

Skobelev, P., Glaschenko, A., Grachev, I. and Inozemtsev, S. (2007), "MAGENTA technology case studies of magenta i-scheduler for road transportation", Proceedings of the 6th International Joint Conference on Autonomous Agents and Multiagent Systems, ACM, May, p. 273.

Smith, S.G., Cortellessa, D., Hildum, D. and Ohler, C.M. (2005), "Using a scheduling domain ontology to compute user-oriented explanations", in Castillo, L., Borrajo, D., Salido, M. and ODDI, A. (Eds), Planning, Scheduling and Constraint Satisfaction: From Theory to Practice, IOS Press, Amsterdam. 
Song, J. and Regan, A. (2004), "An auction based collaborative carrier network. CD-ROM", Vol. 4, Transportation Research Board of the National Academies, Washington, DC, p. 5.

Sprenger, R. and Mönch, L. (2011), "iCoMAS: an agent-based system for cooperative transportation planning in the food industry", Proceedings of the International Conference on Industrial Applications of Holonic and Multi-Agent Systems, pp. 175-184.

Ulku, M.A. (2012), "Dare to care: shipment consolidation reduces not only costs, but also environmental damage", International Journal of Production Economics, Vol. 139 No. 2, pp. 438-446.

Verdonck, L., Caris, A., Ramaekers, K. and Janssens, G.K. (2013), "Collaborative logistics from the perspective of road transportation companies", Transport Reviews, Vol. 33 No. 6, pp. 700-719.

Wagner, M. and Meyr, H. (2015), "Food and beverages", Supply Chain Management and Advanced Planning, Springer, pp. 391-407.

Wang, X. and Kopfer, H. (2014), "Collaborative transportation planning of less-than-truckload freight", OR Spectrum, Vol. 36 No. 2, pp. 357-380.

Yao, E. and Song, Y. (2013), "Study on eco-route planning algorithm and environmental impact assessment", Journal of Intelligent Transportation Systems, Vol. 17 No. 1, pp. 42-53.

\section{Corresponding author}

Muhammad Ali Memon can be contacted at: muhammad.ali@usindh.edu.pk 
This article has been cited by:

1. ZhongDr Ray Y., Dr Ray Y. Zhong, TanProfessor Kim, Professor Kim Tan, BhaskaranProfessor Gopalakrishnan, Professor Gopalakrishnan Bhaskaran. 2017. Data-driven food supply chain management and systems. Industrial Management \& Data Systems 117:9, 1779-1781. [Citation] [Full Text] [PDF] 